\title{
On Primary Ideals. Part I
}

\author{
Yasushige Watase \\ Suginami-ku Matsunoki \\ 3-21-6 Tokyo, Japan
}

\begin{abstract}
Summary. We formalize in the Mizar System [3, 4], definitions and basic propositions about primary ideals of a commutative ring along with Chapter 4 of [1] and Chapter III of [8. Additionally other necessary basic ideal operations such as compatibilities taking radical and intersection of finite number of ideals are formalized as well in order to prove theorems relating primary ideals. These basic operations are mainly quoted from Chapter 1 of [1] and compiled as preliminaries in the first half of the article.
\end{abstract}

MSC: 13A70 16D70 68V20

Keywords: primary ideal; radical ideal; prime ideal

MML identifier: IDEAL_2, version: 8.1.11 5.66.1402

From now on $R$ denotes a commutative ring, $A$ denotes a non degenerated, commutative ring, $I, J, \mathfrak{p}$ denote ideals of $A, \mathfrak{q}$ denotes a prime ideal of $A$, and $M, M_{1}, M_{2}$ denote ideals of $A_{/ \mathfrak{p}}$.

Let us consider $A$ and $\mathfrak{p}$. We introduce the notation $\pi_{A \rightarrow A / \mathfrak{p}}$ as a synonym of the canonical homomorphism of $\mathfrak{p}$ into quotient field.

Now we state the proposition:

(1) Let us consider ideals $a, b$ of $A$, and a prime ideal $\mathfrak{q}$ of $A$. If $a \cap b \subseteq \mathfrak{q}$, then $a \subseteq \mathfrak{q}$ or $b \subseteq \mathfrak{q}$.

Let us consider $A$. Let $a$ be a non empty finite sequence of elements of Ideals $A$ and $i$ be an element of $\operatorname{dom} a$. Let us observe that the functor $a(i)$ yields a non empty subset of $A$. One can check that $a(i)$ is closed under addition, left and right ideal as a subset of $A$ and $\bigcap \operatorname{rng} a$ is closed under addition, left and right ideal as a subset of $A$.

Now we state the proposition:

(2) [1, P.8, Prop. 1.11 II)]:

Let us consider a non empty finite sequence $a$ of elements of Ideals $A$, and 
a prime ideal $\mathfrak{q}$ of $A$. Suppose $\bigcap \operatorname{rng} a \subseteq \mathfrak{q}$. Then there exists an object $i$ such that

(i) $i \in \operatorname{dom} a$, and

(ii) $a(i) \subseteq \mathfrak{q}$.

Proof: Define $\mathcal{P}$ [natural number] $\equiv$ for every non empty finite sequence $a$ of elements of Ideals $A$ for every prime ideal $\mathfrak{q}$ of $A$ such that len $a=\$_{1}$ holds if $\bigcap \operatorname{rng} a \subseteq \mathfrak{q}$, then there exists an object $i$ such that $i \in \operatorname{dom} a$ and $a(i) \subseteq \mathfrak{q}$. For every non zero natural number $n$ such that $\mathcal{P}[n]$ holds $\mathcal{P}[n+1]$. For every non zero natural number $i, \mathcal{P}[i]$.

Let us consider $A$. Let $I$ be an ideal of $A$. The functor $\% I$ yielding a function

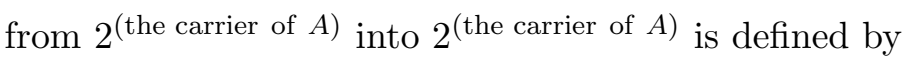

(Def. 1) for every subset $x$ of $A$, it $(x)=x \% I$.

Now we state the propositions:

(3) Let us consider a proper ideal $I$ of $A$, and a non empty finite sequence $F$ of elements of Ideals $A$. Then

(i) $\operatorname{rng}(\% I) \cdot F \neq \emptyset$, and

(ii) $\operatorname{rng} F \neq \emptyset$, and

(iii) $\bigcap \operatorname{rng}(\% I) \cdot F \subseteq$ the carrier of $A$.

(4) [1, P.8, Ex.1.12. IV)]:

Let us consider a proper ideal $I$ of $A$, and a non empty finite sequence $F$ of elements of Ideals $A$. Then $(\% I)(\bigcap \operatorname{rng} F)=\bigcap \operatorname{rng}(\% I) \cdot F$.

Proof: $\operatorname{rng}(\% I) \cdot F \neq \emptyset$. For every object $x$ such that $x \in(\% I)(\bigcap \operatorname{rng} F)$ holds $x \in \bigcap \operatorname{rng}(\% I) \cdot F . \bigcap \operatorname{rng}(\% I) \cdot F \subseteq(\% I)(\bigcap \operatorname{rng} F)$.

(5) $I * \Omega_{A}=I$.

(6) Let us consider finite sequences $f, g$ of elements of $2^{\alpha}$. Suppose len $f \geqslant$ len $g>0$ and $I^{\operatorname{len} f}=f(\operatorname{len} f)$ and $f(1)=I$ and for every natural number $i$ such that $i, i+1 \in \operatorname{dom} f$ holds $f(i+1)=I * f_{/ i}$ and $I^{\text {len } g}=g(\operatorname{len} g)$ and $g(1)=I$ and for every natural number $i$ such that $i, i+1 \in \operatorname{dom} g$ holds $g(i+1)=I * g_{/ i}$. Then $f\lceil\operatorname{dom} g=g$, where $\alpha$ is the carrier of $A$.

Proof: Set $f_{1}=f\lceil\operatorname{dom} g$. For every natural number $i$ such that $i, i+1 \in$ dom $f_{1}$ holds $f_{1}(i+1)=I * f_{1 / i} \cdot f_{1}=g$.

(7) Let us consider a natural number $n$. If $n>0$, then $I^{n+1}=I * I^{n}$. The theorem is a consequence of $(6)$.

(8) [1, P.9, Ex.1.13 II)]:

$\sqrt{I}=\sqrt{\sqrt{I}}$.

Proof: For every object $o$ such that $o \in \sqrt{\sqrt{I}}$ holds $o \in \sqrt{I}$. 
(9) [1, P.9, Ex.1.13 III)]:

$\sqrt{I \cap J}=\sqrt{I} \cap \sqrt{J}$.

Proof: For every object $o$ such that $o \in \sqrt{I \cap J}$ holds $o \in \sqrt{I} \cap \sqrt{J}$. $\sqrt{I} \cap \sqrt{J} \subseteq \sqrt{I \cap J}$.

(10) [1, P.9, Ex.1.13 IV)]:

$\sqrt{I}=\Omega_{A}$ if and only if $I=\Omega_{A}$.

Proof: If $\sqrt{I}=\Omega_{A}$, then $I=\Omega_{A}$ by [7, (2)], [2, (19)].

(11) [1, P.9, Ex.1.13 v)]:

$\sqrt{I+J}=\sqrt{\sqrt{I}+\sqrt{J}}$.

Proof: For every object $o$ such that $o \in \sqrt{I+J}$ holds $o \in \sqrt{\sqrt{I}+\sqrt{J}}$.

$\sqrt{\sqrt{I}+\sqrt{J}} \subseteq \sqrt{I+J}$

(12) [1, P.9, Ex.1.13 VI)]:

Let us consider a prime ideal $\mathfrak{q}$ of $A$, and a non zero natural number $n$. Then $\sqrt{\mathfrak{q}^{n}}=\mathfrak{q}$.

Proof: Define $\mathcal{P}$ [natural number $] \equiv \sqrt{\mathfrak{q}^{\$_{1}}}=\mathfrak{q}$. For every non zero natural number $n$ such that $\mathcal{P}[n]$ holds $\mathcal{P}[n+1]$. For every non zero natural number $i, \mathcal{P}[i]$.

(13) [1, P.9, PRop1.16]:

If $\sqrt{I}$ and $\sqrt{J}$ are co-prime, then $I$ and $J$ are co-prime. The theorem is a consequence of (11) and (10).

(14) Let us consider elements $x, y$ of the carrier of $A / \mathfrak{p}$. Suppose $x, y \in$ $\left(\pi_{A \rightarrow A / \mathfrak{p}}\right)^{\circ} I$. Then $x+y \in\left(\pi_{A \rightarrow A / \mathfrak{p}}\right)^{\circ} I$.

(15) Let us consider elements $a, x$ of the carrier of $A / \mathfrak{p}$. Suppose $x \in\left(\pi_{A \rightarrow A / \mathfrak{p}}\right)^{\circ} I$. Then $a \cdot x \in\left(\pi_{A \rightarrow A / \mathfrak{p}}\right)^{\circ} I$.

(16) $\left(\pi_{A \rightarrow A / \mathfrak{p}}\right)^{\circ} I$ is an ideal of $A / \mathfrak{p}$. The theorem is a consequence of (14) and (15).

(17) Let us consider elements $x, y$ of the carrier of $A$. Suppose $x, y \in$ $\left(\pi_{A \rightarrow A / \mathfrak{p}}\right)^{-1}\left(M_{1}\right)$. Then $x+y \in\left(\pi_{A \rightarrow A / \mathfrak{p}}\right)^{-1}\left(M_{1}\right)$.

(18) Let us consider elements $r, x$ of the carrier of $A$.

Suppose $x \in\left(\pi_{A \rightarrow A / \mathfrak{p}}\right)^{-1}\left(M_{1}\right)$. Then $r \cdot x \in\left(\pi_{A \rightarrow A / \mathfrak{p}}\right)^{-1}\left(M_{1}\right)$.

(19) $\left(\pi_{A \rightarrow A / \mathfrak{p}}\right)^{-1}\left(M_{1}\right)$ is an ideal of $A$. The theorem is a consequence of (17) and (18).

(20) $\mathfrak{p} \subseteq\left(\pi_{A \rightarrow A / \mathfrak{p}}\right)^{-1}\left(M_{1}\right)$.

PROOF: For every object $x$ such that $x \in \mathfrak{p}$ holds $x \in\left(\pi_{A \rightarrow A / \mathfrak{p}}\right)^{-1}\left(M_{1}\right)$ by [5, (13)].

(21) $\left(\pi_{A \rightarrow A / \mathfrak{p}}\right)^{\circ}\left(\left(\pi_{A \rightarrow A / \mathfrak{p}}\right)^{-1}\left(M_{1}\right)\right)=M_{1}$. 
(22) If $\mathfrak{p} \subseteq I$, then $\left(\pi_{A \rightarrow A / \mathfrak{p}}\right)^{-1}\left(\left(\pi_{A \rightarrow A / \mathfrak{p}}\right)^{\circ} I\right)=I$.

Proof: $\left(\pi_{A \rightarrow A / \mathfrak{p}}\right)^{-1}\left(\left(\pi_{A \rightarrow A / \mathfrak{p}}\right)^{\circ} I\right) \subseteq I$.

(23) If $I \subseteq J$, then $\left(\pi_{A \rightarrow A / \mathfrak{p}}\right)^{\circ} I \subseteq\left(\pi_{A \rightarrow A / \mathfrak{p}}\right)^{\circ} J$.

(24) If $M_{1} \subseteq M_{2}$, then $\left(\pi_{A \rightarrow A / \mathfrak{p}}\right)^{-1}\left(M_{1}\right) \subseteq\left(\pi_{A \rightarrow A / \mathfrak{p}}\right)^{-1}\left(M_{2}\right)$.

(25) $\left(\pi_{A \rightarrow A / \mathfrak{p}}\right)^{-1}\left(\Omega_{A_{\mathfrak{p}}}\right)=\Omega_{A}$.

(26) If $M$ is proper, then $\left(\pi_{A \rightarrow A / \mathfrak{p}}\right)^{-1}(M)$ is proper. The theorem is a consequence of (21).

(27) If $\mathfrak{p} \subseteq I$ and $I$ is maximal, then $\left(\pi_{A \rightarrow A / \mathfrak{p}}\right)^{\circ} I$ is maximal. The theorem is a consequence of (16), (25), (22), (26), (19), and (24).

Let us consider $A$ and $\mathfrak{p}$. The functor $\Psi_{\mathfrak{p}}$ yielding a function from Ideals $A / \mathfrak{p}$ into $\operatorname{Ideals}(A, \mathfrak{p})$ is defined by

(Def. 2) for every element $x$ of Ideals $A / \mathfrak{p}, i t(x)=\left(\pi_{A \rightarrow A / \mathfrak{p}}\right)^{-1}(x)$.

Let $J$ be a proper ideal of $A$. Observe that $A / J$ is non degenerated and commutative.

[1, p.2, Prop. 1.1]:

Let us consider $A$. Let $\mathfrak{p}$ be an ideal of $A$. Let us note that $\Psi_{\mathfrak{p}}$ is one-to-one and $\subseteq$-monotone.

[1. p.50, Chapter 4]:

Let $A$ be a well unital, non empty double loop structure and $S$ be a subset of $A$. We say that $S$ is quasi-primary if and only if

(Def. 3) for every elements $x, y$ of $A$ such that $x \cdot y \in S$ holds $x \in S$ or $y \in \sqrt{S}$.

We say that $S$ is primary if and only if

(Def. 4) $S$ is proper and quasi-primary.

Let $K$ be a well unital, non empty double loop structure. Let us note that every subset of $K$ which is primary is also proper and quasi-primary and every subset of $K$ which is proper and quasi-primary is also primary.

Now we state the proposition:

(28) Let us consider an ideal $\mathfrak{q}$ of $A$. If $\mathfrak{q}$ is prime, then $\mathfrak{q}$ is primary.

Proof: For every elements $x, y$ of $A$ such that $x \cdot y \in \mathfrak{q}$ holds $x \in \mathfrak{q}$ or $y \in \sqrt{\mathfrak{q}}$.

Let us consider $A$. One can verify that every ideal of $A$ which is prime is also primary.

Let $A$ be a non degenerated, commutative ring. Let us observe that there exists a proper ideal of $A$ which is primary.

Now we state the propositions:

(29) $\quad I$ is primary if and only if $I \neq \Omega_{A}$ and for every elements $x, y$ of $A$ such that $x \cdot y \in I$ and $x \notin I$ holds $y \in \sqrt{I}$. 
(30) $I \neq \Omega_{A}$ and for every elements $x, y$ of $A$ such that $x \cdot y \in I$ and $x \notin I$ holds $y \in \sqrt{I}$ if and only if $A / I$ is not degenerated and for every element $z$ of $A / I$ such that $z$ is zero-divisible holds $z$ is nilpotent.

Proof: If $I \neq \Omega_{A}$ and for every elements $x, y$ of $A$ such that $x \cdot y \in I$ and $x \notin I$ holds $y \in \sqrt{I}$, then $A / I$ is not degenerated and for every element $z$ of $A / I$ such that $z$ is zero-divisible holds $z$ is nilpotent. If $A / I$ is not degenerated and for every element $z$ of $A / I$ such that $z$ is zero-divisible holds $z$ is nilpotent, then $I \neq \Omega_{A}$ and for every elements $x_{1}, y_{1}$ of $A$ such that $x_{1} \cdot y_{1} \in I$ and $x_{1} \notin I$ holds $y_{1} \in \sqrt{I}$ by [6, (2)].

(31) $I$ is primary if and only if $A / I$ is not degenerated and for every element $x$ of $A / I$ such that $x$ is zero-divisible holds $x$ is nilpotent. The theorem is a consequence of (29) and (30).

[1, p.50, Prop. 4.1]:

Let us consider $A$. Let $Q$ be a primary ideal of $A$. Note that $\sqrt{Q}$ is prime.

Let $I$ be a primary ideal of $A$. One can verify that every element of $A / I$ which is zero-divisible is also nilpotent.

Let $P, Q$ be ideals of $A$. We say that $Q$ is $P$-primary if and only if

(Def. 5) $\sqrt{Q}=P$.

The functor PrimaryIdeals $(A)$ yielding a family of subsets of the carrier of $A$ is defined by the term

(Def. 6) the set of all $I$ where $I$ is a primary ideal of $A$.

Note that PrimaryIdeals $(A)$ is non empty.

Let us consider $\mathfrak{q}$. The functor PrimaryIdeals $(A, \mathfrak{q})$ yielding a non empty subset of PrimaryIdeals $(A)$ is defined by the term

(Def. 7) $\{I$, where $I$ is a primary ideal of $A: I$ is $\mathfrak{q}$-primary $\}$.

Let us consider a proper ideal $\mathfrak{p}$ of $A$. Now we state the propositions:

(32) $\left(\pi_{A \rightarrow A / \mathfrak{p}}\right)^{\circ} \sqrt{\mathfrak{p}}=\operatorname{nilrad}(A / \mathfrak{p})$.

Proof: For every object $x$ such that $x \in \operatorname{nilrad}(A / \mathfrak{p})$ holds $x \in\left(\pi_{A \rightarrow A / \mathfrak{p}}\right)^{\circ} \sqrt{\mathfrak{p}}$. $\left(\pi_{A \rightarrow A / \mathfrak{p}}\right)^{\circ} \sqrt{\mathfrak{p}} \subseteq \operatorname{nilrad}(A / \mathfrak{p})$.

(33) If $\sqrt{\mathfrak{p}}$ is maximal, then $A / \mathfrak{p}$ is local.

ProOF: Set $m=\sqrt{\mathfrak{p}} \cdot\left(\pi_{A \rightarrow A / \mathfrak{p}}\right)^{\circ} m=\operatorname{nilrad}(A / \mathfrak{p})$. For every objects $m_{1}$, $m_{2}$ such that $m_{1}, m_{2} \in \mathrm{m}-\operatorname{Spectrum}(A / \mathfrak{p})$ holds $m_{1}=m_{2}$.

(34) [1, P.51, PROP. 4.2]:

Let us consider a proper ideal $\mathfrak{p}$ of $A$. If $\sqrt{\mathfrak{p}}$ is maximal, then $\mathfrak{p}$ is primary. Proof: Set $m=\sqrt{\mathfrak{p}}$. $\left(\pi_{A \rightarrow A / \mathfrak{p}}\right)^{\circ} m$ is maximal. $A / \mathfrak{p}$ is local. For every element $x$ of $A / \mathfrak{p}$ such that $x$ is zero-divisible holds $x$ is nilpotent. 
(35) [1, P.51, Prop. 4.2] CASE of M is maximal Ideal:

Let us consider a maximal ideal $M$ of $A$, and a non zero natural number $n$. Then $M^{n} \in \operatorname{PrimaryIdeals}(A, M)$. The theorem is a consequence of (12) and (34).

(36) Let us consider ideals $q_{1}, q_{2}$ of $A$. Suppose $q_{1}, q_{2} \in \operatorname{PrimaryIdeals}(A, \mathfrak{q})$. Then $q_{1} \cap q_{2} \in \operatorname{PrimaryIdeals}(A, \mathfrak{q})$. The theorem is a consequence of $(9)$ and (29).

(37) [1, P.51, Lemma 4.3]:

Let us consider a prime ideal $\mathfrak{q}$ of $A$, and a non empty finite sequence $F$ of elements of $\operatorname{PrimaryIdeals}(A, \mathfrak{q})$. Then $\bigcap \operatorname{rng} F \in \operatorname{PrimaryIdeals}(A, \mathfrak{q})$. Proof: $\bigcap \operatorname{rng} F \in \operatorname{Primary} \operatorname{Ideals}(A, \mathfrak{q})$.

(38) Let us consider a proper ideal $I$ of $A$, and an element $x$ of $\sqrt{I}$. Then there exists a natural number $m$ such that

(i) $m \in\left\{n\right.$, where $n$ is an element of $\left.\mathbb{N}: x^{n} \notin I\right\}$, and

(ii) $x^{m+1} \in I$.

Proof: Consider $x_{1}$ being an element of $A$ such that $x_{1}=x$ and there exists an element $n$ of $\mathbb{N}$ such that $x_{1}{ }^{n} \in I$. Consider $n_{1}$ being an element of $\mathbb{N}$ such that $x_{1}{ }^{n_{1}} \in I$. $n_{1} \notin\left\{n\right.$, where $n$ is an element of $\left.\mathbb{N}: x^{n} \notin I\right\}$. $0 \in\left\{n\right.$, where $n$ is an element of $\left.\mathbb{N}: x^{n} \notin I\right\}$. $\{n$, where $n$ is an element of $\left.\mathbb{N}: x^{n} \notin I\right\}=\mathbb{N}$.

(39) Let us consider proper ideals $I, J$ of $A$. Suppose $I \subseteq J \subseteq \sqrt{I}$ and for every elements $x, y$ of $A$ such that $x \cdot y \in I$ and $x \notin I$ holds $y \in J$. Then

(i) $I$ is primary, and

(ii) $\sqrt{I}=J$, and

(iii) $J$ is prime.

Proof: $\sqrt{I} \subseteq J$.

(40) Let us consider a proper ideal $Q$ of $A$. Suppose for every elements $x, y$ of $A$ such that $x \cdot y \in Q$ and $y \notin \sqrt{Q}$ holds $x \in Q$. Then

(i) $Q$ is primary, and

(ii) $\sqrt{Q}$ is prime.

The theorem is a consequence of (39).

(41) [1, P.51, LEMMA 4.4 I)]:

Let us consider an ideal $\mathfrak{p}$ of $A$, and an element $x$ of $A$. Suppose $x \in \mathfrak{p}$. Then $\mathfrak{p} \%\{x\}$-ideal $=\Omega_{A}$.

Proof: Set $I=\{x\}$-ideal. If $x \in \mathfrak{p}$, then $\mathfrak{p} \% I=\Omega_{A}$. 
Let us consider an ideal $\mathfrak{p}$ of $A$, and an element $x$ of $A$. Suppose $\mathfrak{p} \in$ PrimaryIdeals $(A, \mathfrak{q})$. If $x \notin \mathfrak{p}$, then $\mathfrak{p} \%\{x\}$-ideal $\in \operatorname{PrimaryIdeals}(A, \mathfrak{q})$. Proof: Set $I=\{x\}$-ideal. Consider $q_{1}$ being a primary ideal of $A$ such that $q_{1}=\mathfrak{p}$ and $q_{1}$ is $\mathfrak{q}$-primary. If $x \notin \mathfrak{p}$, then $\mathfrak{p} \% I \in \operatorname{PrimaryIdeals}(A, \mathfrak{q})$.

(43) [1, P.51, LEMma 4.4 III)]:

Let us consider an ideal $\mathfrak{p}$ of $A$, and an element $x$ of $A$. Suppose $\mathfrak{p} \in$ PrimaryIdeals $(A, \mathfrak{q})$. If $x \notin \mathfrak{q}$, then $\mathfrak{p} \%\{x\}$-ideal $=\mathfrak{p}$.

Proof: Set $I=\{x\}$-ideal. Consider $Q$ being a primary ideal of $A$ such that $Q=\mathfrak{p}$ and $Q$ is $\mathfrak{q}$-primary. If $x \notin \mathfrak{q}$, then $\mathfrak{p} \% I=\mathfrak{p}$.

\section{REFERENCES}

[1] Michael Francis Atiyah and Ian Grant Macdonald. Introduction to Commutative Algebra, volume 2. Addison-Wesley Reading, 1969.

[2] Jonathan Backer, Piotr Rudnicki, and Christoph Schwarzweller. Ring ideals Formalized Mathematics, 9(3):565-582, 2001.

[3] Grzegorz Bancerek, Czesław Byliński, Adam Grabowski, Artur Korniłowicz, Roman Matuszewski, Adam Naumowicz, Karol Pąk, and Josef Urban. Mizar: State-of-the-art and beyond. In Manfred Kerber, Jacques Carette, Cezary Kaliszyk, Florian Rabe, and Volker Sorge, editors, Intelligent Computer Mathematics, volume 9150 of Lecture Notes in Computer Science, pages 261-279. Springer International Publishing, 2015. ISBN 978-3319-20614-1. doi $10.1007 / 978-3-319-20615-8 \_17$.

[4] Grzegorz Bancerek, Czesław Byliński, Adam Grabowski, Artur Korniłowicz, Roman Matuszewski, Adam Naumowicz, and Karol Pak. The role of the Mizar Mathematical Library for interactive proof development in Mizar. Journal of Automated Reasoning, 61(1):9-32, 2018. do1:10.1007/s10817-017-9440-6

[5] Artur Korniłowicz and Christoph Schwarzweller. The first isomorphism theorem and other properties of rings. Formalized Mathematics, 22(4):291-301, 2014. doi 10.2478/forma-20140029 .

[6] Christoph Schwarzweller. On roots of polynomials over $F[X] /\langle p\rangle$. Formalized Mathematics, 27(2):93-100, 2019. doi 10.2478/forma-2019-0010.

[7] Yasushige Watase. Zariski topology. Formalized Mathematics, 26(4):277-283, 2018. doi:10.2478/forma-2018-0024.

[8] Oscar Zariski and Pierre Samuel. Commutative Algebra I. Springer, 2nd edition, 1975.

Accepted June 30, 2021 